\title{
Sintomas de Depressão, Ansiedade e Estresse em Estudantes de Medicina e Estratégias Institucionais de Enfrentamento
}

\section{Symptoms of Depression, Anxiety and Stress in Medical Students and Institutional Coping Strategies}

\author{
Deyvison Soares da Costa ${ }^{1}$ (iD \\ Natany de Souza Batista Medeiros ${ }^{\mathrm{I}}(\mathrm{D})$ \\ Rayane Alves Cordeiro ${ }^{\mathrm{I}} \mathrm{iD}$ \\ Everton de Souza Frutuoso ${ }^{\mathrm{I}}$ (D) \\ Johnnatas Mikael Lopes II $^{\text {ID }}$ \\ Simone da Nóbrega Tomaz Moreira ${ }^{\mathrm{I}}$ (C)
}

\section{PALAVRAS-CHAVE}

- Educação Médica.

- Saúde Mental.

- Estresse Psicológico

- Ansiedade

- Depressão.
${ }^{1}$ Universidade Federal do Rio Grande do Norte, Natal, Rio Grande do Norte, Brasil.

${ }^{\mathrm{II}}$ Universidade Federal do Vale do São Francisco, Paulo Afonso, Bahia, Brasil.

Introdução: Este estudo teve como propósito estimar a prevalência de sintomas de estresse, depressão e ansiedade dos estudantes de Medicina da Universidade Federal do Rio Grande do Norte (UFRN), associando-os com outros fatores. Métodos: Trata-se de um estudo quantitativo epidemiológico, do tipo transversal. Participaram desta pesquisa estudantes de Medicina da UFRN, distribuídos equitativamente entre os diferentes períodos do curso. Entregaram-se 288 questionários, no entanto validaram-se 279, os demais não foram respondidos corretamente. Este estudo foi aprovado pelo Comitê de Ética em Pesquisa da UFRN (Parecer $n^{\circ}$ 2.009.026) e todos os voluntários assinaram o Termo de Consentimento Livre e Esclarecido (TCLE), após serem informados sobre os objetivos da pesquisa. Para a coleta dos dados, utilizaram-se os seguintes instrumentos: 1. ficha de Identificação para os dados sociodemográficos, 2. Inventário de Sintomas de Stress para Adultos de Lipp (ISSL), 3. Inventário de Depressão de Beck (IDB) e 4. Inventário de Ansiedade de Beck (BAI). Para análise dos resultados, realizaram-se análises descritivas a partir da média, do desvio padrão e da frequência dos dados coletados. A análise inferencial foi realizada para verificar a associação entre as variáveis de desfechos $e$ os atributos dos estudantes. Resultados: Dos estudantes entrevistados, 66,3\% tinham estresse e a maioria estava na fase de resistência (58,4\%) com predominância de sintomas psicológicos (42,3\%). Em relação à sintomatologia depressiva, $28 \%$ dos estudantes apresentavam sintomas: $51,3 \%$ com sinais de depressão de leve a moderada, 35,9\% com sinais de depressão moderada e 12,8\% com sinais de depressão severa. No que se refere à sintomatologia ansiosa, 66,3\% dos estudantes apresentavam sinais de ansiedade em seu grau mínimo; e 33,7\%, sinais de ansiedade leve, moderada ou severa. Desses últimos, 21,9\% tinham sinais de ansiedade leve; 10,8\%, sinais de ansiedade moderada; e 1\%, sinais de ansiedade severa. Conclusões: Diante disso, acredita-se que as instituições de ensino superior devem se comprometer com o desenvolvimento integral dos seus estudantes apresentando estratégias institucionais para o enfrentamento dessa realidade. 


\section{KEYWORDS}

- Medical Education.

- Mental Health.

- Psychological Stress.

- Anxiety.

- Depression.

Recebido em 14/3/19

Aceito em 20/10/19

\section{INTRODUÇÃO}

Estudos apontam que estudantes universitários estão mais suscetíveis à manifestação de transtornos mentais que a população geral ${ }^{1}$. A presença desses transtornos mentais, entre estudantes universitários, pode interferir no seu bem-estar psicossocial, nas suas relações interpessoais, como também no seu desempenho acadêmico².

O estudante universitário brasileiro começa sua formação no final da adolescência e início da vida adulta, período que se caracteriza por mudanças psicossociais importantes, como a aquisição de uma nova identidade, inclusive com uma maior autonomia sobre a sua vida e as suas escolhas. Além disso, para alguns estudantes, entrar na universidade significa sair de casa, morar em outra cidade ou até mesmo em outro estado, precisando enfrentar o dilema da separação parental e familiar. Isso implica responsabilizar-se pelos afazeres domésticos, tendo que cozinhar, arrumar casa, administrar os recursos financeiros, entre outras atribuições ${ }^{3}$.

Acrescidos a essas mudanças, existem os vários fatores inerentes à formação médica, que podem contribuir para o estresse desses estudantes, como: carga horária intensa e extensa, dificuldades em conciliar vida pessoal e acadêmica, competitividade entre os estudantes, privação do sono, realização de exame físico em pacientes e medo de adquirir doenças e de cometer erros ${ }^{4}$. Além disso, o contexto do curso médico aproxima os estudantes de situações de dor, sofrimento e morte, e, na maioria dos casos, não há ações institucionais que possam levá-los a refletir sobre os sentimentos advindos do contato com o paciente, até porque a racionalidade científica, presente na formação médica, não valoriza aspectos subjetivos do cotidiano acadêmico ${ }^{5}$.

Essas características inerentes ao estudante universitário e à formação médica podem deixá-lo mais vulnerável à manifestação de sofrimento

\section{ABSTRACT}

Introduction: The purpose of this study was to estimate the prevalence of stress, depression and anxiety symptoms among medical students of the Federal University of Rio Grande do Norte, associating them with other factors. Methods: This is a quantitative cross-sectional epidemiological study. Medical students from the Federal University of Rio Grande do Norte participated in this study, distributed equally among the different periods of the course. 288 questionnaires were delivered; however, 279 were validated, while the others were not answered correctly. This study was approved by the Research Ethics Committee of UFRN (Opinion $n$. $2,009,026)$ and all volunteers signed the free and informed consent (FIC) form, after being informed about the research objectives. The following instruments were used to collect the data: (1) Identification card for the socio demographic data, (2) Inventory of Stress Symptoms for Adults of Lipp (ISSL), (3) Beck Depression Inventory BDI, (4) Beck Anxiety Inventory (BAI). For the analysis of the results, descriptive analyzes were performed based on the mean, standard deviation and frequency of the collected data. Inferential analysis was performed between outcome variables and student attributes. Results: Of the interviewed students, a predominance of psychological symptoms (42.3\%). In relation to depressive symptomatology, 28\% of the students had symptoms, with 51.3\% showing signs of mild to moderate depression, 35.9\% signs of moderate depression and $12.8 \%$ showing signs of severe depression. Regarding the anxious symptomatology, 66.3\% of the students showed minimal signs of anxiety and 33.7\% showed signs of mild, moderate or severe anxiety. Of the latter, $21.9 \%$ had signs of mild anxiety, $10.8 \%$ signs of moderate anxiety and $1 \%$ signs of severe anxiety. Conclusions: In view of this, it is believed that higher education institutions should commit themselves to the integral development of their students, offering institutional strategies for students to cope with this reality. 
adequadas à resolução dos problemas característicos da profissão.

Diante do exposto, este estudo teve como propósito estimar a prevalência de sintomas de estresse, depressão e ansiedade dos estudantes de Medicina da Universidade Federal do Rio Grande do Norte (UFRN), associando-os com outros fatores.

\section{METODOLOGIA}

Trata-se de um estudo quantitativo epidemiológico, do tipo transversal. O estudo foi aprovado pelo Comitê de Ética em Pesquisa da UFRN (Parecer $\mathrm{n}^{\circ}$ 2.009.026) e todos os voluntários assinaram o Termo de Consentimento Livre e Esclarecido (TCLE), após serem informados sobre os objetivos da pesquisa.

Realizou-se o estudo na UFRN, no câmpus central. A coleta dos dados foi feita no período de maio a junho de 2017.

Para a estimativa amostral, utilizaram-se um intervalo de confiança de $95 \%(z=1,96)$, um erro de amostra de $5 \%$ e uma prevalência de transtornos mentais em universitários estimada em 25\%. Assim, a amostra compreendeu um total de 288 estudantes do curso de Medicina da instituição em questão, regularmente matriculados, escolhidos aleatoriamente e distribuídos equitativamente entre os diferentes períodos do curso.

Para caracterização da população do estudo, utilizou-se uma ficha de identificação para os dados sociodemográficos, que incluía questões sobre idade, sexo, estado civil, religião, renda familiar mensal, estado de origem, situação de moradia, ensino fundamental, ensino médio e período em que se encontra no curso. Os estudantes também foram questionados sobre a atuação em ligas acadêmicas, a participação em grupos de pesquisa, atividades extracurriculares, a percepção da cobrança do curso, a carga horária, a preparação para o mercado de trabalho, a prática de atividade física, os momentos de lazer, o uso de drogas, o atendimento psicológico/ psiquiátrico prévio e horas de sono.

Para conhecer a prevalência de sintomas depressivos entre os estudantes de Medicina da UFRN, adotou-se o Inventário de Depressão de Beck (IDB), instrumento de rastreamento de sintomas depressivos. Tratase um questionário autoaplicativo que foi traduzido para vários idiomas e validado em diferentes países, inclusive no Brasil ${ }^{12}$. A escala consiste em 21 itens referentes a tristeza, pessimismo, sensação de fracasso, falta de satisfação, sensação de culpa, sensação de punição, entre outros. O IDB possui quatro categorias de classificação dos sintomas depressivos para amostras não diagnosticadas, subdivididas por escores: 0-15 (nenhum ou mínimo), 16-20 (leve), 21-29 (moderado) e 30-63 (grave) ${ }^{13}$.

Em relação à avaliação dos sinais e sintomas da ansiedade na população estudada, lançou-se mão do Inventário de Ansiedade de Beck, em inglês Beck Anxiety Inventory (BAI). Traduzido e validado no Brasil por Cunha em 2001, o BAI consiste em 21 itens que refletem somática, afetiva e cognitivamente os sintomas da ansiedade ${ }^{14,15}$. Cada um desses itens contém quatro alternativas, que representam níveis de gravidade crescente de cada sintoma, os quais variam de 0 a 3 . O escore total (pontuação) pode variar de 0 a 63, o que possibilita a classificação em níveis de intensidade dos sintomas da ansiedade. Para interpretação da ansiedade autoinformada, adotam-se os seguintes pontos de corte: escore de 0 a 10 - sinais mínimos de ansiedade; de 11 a 19 - sinais de ansiedade leve; de 20 a 30 - sinais de ansiedade moderada; e de 31 a 63 - sinais de ansiedade grave ${ }^{15,16}$.
Com o objetivo de avaliar o estresse dos estudantes, utilizou-se o Inventário de Sintomas de Stress para Adultos de Lipp (ISSL) ${ }^{17}$, validado em 1994 por Lipp e Guevara. Adotado em dezenas de pesquisas e trabalhos clínicos na área do estresse, o ISSL fornece uma medida objetiva da sintomatologia do estresse em jovens acima de 15 anos e adultos ${ }^{18,19}$. Essa ferramenta avalia os sintomas físicos e psicológicos experimentados nas últimas 24 horas, na última semana e no último mês, e permite estabelecer um diagnóstico preciso sobre a ocorrência do estresse e a fase em que se encontra (alerta, resistência, quase exaustão e exaustão), além de demonstrar se a sintomatologia é predominante física ou psicológica. No total, o ISSL apresenta 37 itens de natureza somática e 19 referentes aos aspectos psicológicos, já que os sintomas muitas vezes se repetem, diferindo somente na intensidade e seriedade ${ }^{20}$. A aplicação do ISSL é prática e fácil, e a correção é feita de acordo com as instruções do Manual do Inventário de Sintomas de Stress para Adultos de Lipp (ISSL) ${ }^{17}$.

Realizaram-se análises descritivas a partir da média, do desvio padrão e da frequência dos dados coletados. A análise inferencial foi feita para verificar a associação entre as variáveis de desfechos e os atributos dos estudantes (sexo, renda, religião, estado de origem, ensino fundamental, ensino médio, período em que se encontra no curso, participação em ligas acadêmicas, grupos de pesquisa, atividades extracurriculares, a percepção da cobrança do curso, a carga horária, a prática de atividade física, os momentos de lazer, o uso de drogas, atendimento psicológico/psiquiátrico prévio e horas de sono). Como medida de efeito para estimar a associação com o desfecho, adotou-se a razão de prevalência (RP) ajustada a partir da teoria dos modelos lineares generalizados. Foram realizados modelos com três variáveis de desfecho: grau de sintomas depressivos, grau de sintomas ansiosos e presença de sintomas de exaustão. Como se trata de uma pesquisa transversal, adotou-se uma distribuição de Poisson com função ligante log-linear e matriz de covariância robusta. Adotou-se um nível de significância de 5\%.

\section{RESULTADOS}

Dos 288 questionários respondidos, validaram-se 279 , e os demais não foram preenchidos corretamente. Os estudantes apresentaram média de 22,9 anos ( $\mathrm{DP}=4,29)$. Em sua maioria, eram solteiros $(97,8 \%)$, com renda familiar acima de quatro salários mínimos (77,8\%), 64,5\% viviam com a família e $76,0 \%$ eram do estado do Rio grande do Norte (RN). A maioria cursou o ensino fundamental $(80,3 \%)$ e o ensino médio $(60,6 \%)$ em escolas privadas.

Cerca da metade dos estudantes (51,6\%) não está inserida em ligas acadêmicas nem em grupos de pesquisa científica (58,4\%). A maioria está envolvida em atividades acadêmicas extracurriculares (71,3\%) e 96,8\% dos alunos relatam se sentir cobrados pelo curso. Em geral, não consideram a carga horária do curso adequada $(72,0 \%)$ e não se sentem seguros para a inserção no mercado de trabalho $(68,5 \%)$.

Além disso, em relação ao cotidiano, $61,6 \%$ já fizeram uso de alguma droga lícita ou ilícita, $84,6 \%$ têm momentos de lazer semanal e $62,4 \%$ praticam atividade física regularmente. Os estudantes dormem, em média, seis horas por dia. Pouco mais da metade dos estudantes era do sexo feminino $(50,5 \%)$ e já precisou de algum atendimento psicológico ou psiquiátrico ao longo da vida $(50,5 \%)$.

A Tabela 1 apresenta as porcentagens da presença de sintomas ansiosos, depressivos e de estresse na amostra total e divididos pelo

REVISTA BRASILEIRA DE EDUCAÇC̃̃ MÉDICA

3 44 (1) : e040; 2020 
agrupamento dos períodos em três ciclos: do primeiro ao quarto período, do quinto ao oitavo e do nono ao $12^{\circ}$. De acordo com o ISSL, $185(66,3 \%)$ estudantes apresentaram sinais e sintomas de estresse, sendo dois $(0,7 \%)$ na fase de alerta, $163(58,4 \%)$ na fase de resistência, dez (3,6\%) na fase de quase exaustão e dez $(3,6 \%)$ na fase de exaustão. Os sintomas psíquicos $(42,3 \%)$ mostraram-se como sintomatologia predominante. Pela ANOVA de um fator, não houve diferenças nos sintomas ansiosos $(\mathrm{F}(2)=2,28$, $\mathrm{p}$ $=0,10)$, depressivos $(\mathrm{F}(2)=2,85, \mathrm{p}=0,06)$ e de estresse $(\mathrm{F}(2)=0,37, \mathrm{p}=$ $0,70)$ entre os períodos do curso.

Verificou-se que 78 (28\%) estudantes possuíam sintomatologia depressiva, dos quais $40(51,3 \%)$ com sinais de depressão de leve a moderada, 28 (35,9\%) com sinais de depressão moderada e dez (12,8\%) com sinais de depressão severa. Em relação à sintomatologia ansiosa, 185 $(66,3 \%)$ estudantes apresentaram sinais de ansiedade em seu grau mínimo; e $94(33,7 \%)$, sinais de ansiedade leve, moderada ou severa. Desses últimos, $61(21,9 \%)$ apresentaram sinais de ansiedade leve; 30 (10,8\%); sinais de ansiedade moderada; e três (1\%), sinais de ansiedade severa.

A análise inferencial (Tabelas 2, 3 e 4) revelou que os fatores associados à presença de sintomas depressivos foram: onde estudou o ensino médio $(\mathrm{p}<0,05)$, sentir-se cobrado $(\mathrm{p}<0,05)$, presença de estresse $(\mathrm{p}<0,001)$ e de sintomas de exaustão $(\mathrm{p}<0,001)$ e horas de sono $(\mathrm{p}<0,05)$. Quando se verificaram as análises descritivas desses fatores, constatou-se que os estudantes com graus mais elevados de sintomatologia depressiva haviam estudado o ensino médio em escola pública, sentiam-se cobrados pelo curso, apresentavam estresse e sintomas de exaustão e possuíam menores quantidades de horas de sono.

Em relação à presença de sintomas ansiosos, verificou-se que os fatores associados foram sexo $(\mathrm{p}<0,01)$, atendimento psicológico prévio $(\mathrm{p}<0,05)$, horas de sono $(\mathrm{p}<0,01)$ e período do curso $(\mathrm{p}<0,01)$. Os estudantes que apresentaram graus mais elevados de sintomatologia ansiosa eram do sexo feminino, já haviam feito acompanhamento psicológico, dormiam menos horas e estavam nos primeiros dois ciclos do curso.

A presença de sintomas de exaustão foi associada à realização de atendimento psicológico prévio $(\mathrm{p}<0,05)$, de modo que os estudantes que já haviam passado por atendimento psicológico apresentaram sintomas de exaustão.

\section{Tabela 1}

Porcentagens da presença de sintomas ansiosos, depressivos e de estresse em estudantes de Medicina da UFRN, em 2017, Natal-RN, Brasil, divididos em três ciclos ao longo do curso

\begin{tabular}{|c|c|c|c|c|c|}
\hline \multirow{2}{*}{\multicolumn{2}{|c|}{ Variáveis }} & Total & Do primeiro ao quarto período & Do quinto ao oitavo período & Do nono ao $12^{\circ}$ período \\
\hline & & $\%$ & $\%$ & $\%$ & $\%$ \\
\hline \multirow{2}{*}{ Presença de estresse } & Não & $33,7 \%$ & $31,3 \%$ & $33,7 \%$ & $36,2 \%$ \\
\hline & Sim & $66,3 \%$ & $68,8 \%$ & $66,3 \%$ & $63,8 \%$ \\
\hline \multirow{5}{*}{ Fase do estresse } & Não se aplica & $33,7 \%$ & $31,3 \%$ & $33,7 \%$ & $36,2 \%$ \\
\hline & Alerta & $0,7 \%$ & $1,0 \%$ & $1,1 \%$ & $0,0 \%$ \\
\hline & Resistência & $58,4 \%$ & $59,4 \%$ & $57,3 \%$ & $58,5 \%$ \\
\hline & Quase exaustão & $3,6 \%$ & $2,1 \%$ & $5,6 \%$ & $3,2 \%$ \\
\hline & Exaustão & $3,6 \%$ & $6,3 \%$ & $2,2 \%$ & $2,1 \%$ \\
\hline \multirow{4}{*}{$\begin{array}{l}\text { Predominância do tipo } \\
\text { de sintoma de estresse }\end{array}$} & Não se aplica & $33,7 \%$ & $31,3 \%$ & $33,7 \%$ & $36,2 \%$ \\
\hline & Físico & $17,2 \%$ & $16,7 \%$ & $16,9 \%$ & $18,1 \%$ \\
\hline & Psíquico & $42,3 \%$ & $43,8 \%$ & $42,7 \%$ & $40,4 \%$ \\
\hline & Ambos/empate & $6,8 \%$ & $8,3 \%$ & $6,7 \%$ & $5,3 \%$ \\
\hline \multirow{4}{*}{$\begin{array}{c}\text { Presença de } \\
\text { sintomatologia } \\
\text { depressiva }\end{array}$} & Sem depressão & $72,0 \%$ & $64,6 \%$ & $73,0 \%$ & $78,7 \%$ \\
\hline & De leve a moderada & $14,3 \%$ & $15,6 \%$ & $15,7 \%$ & $11,7 \%$ \\
\hline & Moderada/severa & $10,0 \%$ & $14,6 \%$ & $9,0 \%$ & $6,4 \%$ \\
\hline & Severa & $3,6 \%$ & $5,2 \%$ & $2,2 \%$ & $3,2 \%$ \\
\hline \multirow{4}{*}{$\begin{array}{c}\text { Presença de } \\
\text { sintomatologia ansiosa }\end{array}$} & Grau mínimo & $66,3 \%$ & $59,4 \%$ & $64,0 \%$ & $75,5 \%$ \\
\hline & Ansiedade leve & $21,9 \%$ & $27,1 \%$ & $18,0 \%$ & $20,2 \%$ \\
\hline & Ansiedade moderada & $10,8 \%$ & $12,5 \%$ & $15,7 \%$ & $4,3 \%$ \\
\hline & Ansiedade severa & $1,1 \%$ & $1,0 \%$ & $2,2 \%$ & $0,0 \%$ \\
\hline
\end{tabular}

Fonte: Elaborada pelos autores 


\begin{tabular}{|c|c|c|c|c|c|}
\hline \multirow{3}{*}{ Variáveis } & \multirow{3}{*}{$\%(n)$} & \multicolumn{4}{|c|}{ Modelo ajustado } \\
\hline & & \multirow{2}{*}{ RP } & \multicolumn{2}{|c|}{ IC95\% } & \multirow{2}{*}{ p-valor } \\
\hline & & & Limite inferior & Limite superior & \\
\hline \multicolumn{6}{|l|}{ Sexo } \\
\hline Feminino & $49,6(137)$ & 0,906 & 0,729 & 1,125 & 0,371 \\
\hline Masculino & $50,4(139)$ & 1 & & & \\
\hline \multicolumn{6}{|l|}{ Renda familiar } \\
\hline Acima de um salário & $3,6(10)$ & 1,058 & 0,649 & 1,726 & \multirow{3}{*}{0,145} \\
\hline Acima de três salários & $18,5(51)$ & 1,272 & 0,995 & 1,628 & \\
\hline Acima de quatro salários & $77,9(215)$ & 1 & & & \\
\hline \multicolumn{6}{|l|}{ Com quem reside } \\
\hline Família & $64,9(179)$ & 0,887 & & & \multirow{5}{*}{0,869} \\
\hline Parentes & $7,2(20)$ & 0,941 & 0,661 & 1,19 & \\
\hline Amigos & $13,4(37)$ & 0,915 & 0,639 & 1,385 & \\
\hline Residência universitária & $14,5(40)$ & 1 & 0,669 & 1,252 & \\
\hline Sozinho & & & & & \\
\hline \multicolumn{6}{|l|}{ Estado } \\
\hline RN & $76,4(211)$ & 0,751 & 0,558 & 1,012 & 0,06 \\
\hline Outro estado & $23,6(65)$ & 1 & & & \\
\hline \multicolumn{6}{|l|}{ Ensino médio } \\
\hline Privada & $37,3(103)$ & 1,042 & 0,787 & 1,379 & \multirow{3}{*}{0,027} \\
\hline Pública & $61,2(169)$ & 0,763 & 0,583 & 0,999 & \\
\hline Misto & $1,4(4)$ & 1 & & & \\
\hline \multicolumn{6}{|l|}{ Atendimento psicológico } \\
\hline Sim & $49,6(137)$ & 0,844 & 0,668 & 1,067 & 0,157 \\
\hline Não & $50,4(139)$ & 1 & & & \\
\hline \multicolumn{6}{|l|}{ Lazer semanal } \\
\hline Sim & $15,6(43)$ & 1,203 & 0,953 & 1,518 & 0,12 \\
\hline Não & $84,4(233)$ & 1 & & & \\
\hline \multicolumn{6}{|l|}{ Atividade física } \\
\hline Sim & $38(105)$ & 1,067 & 0,859 & 1,326 & 0,556 \\
\hline Não & $62(171)$ & 1 & & & \\
\hline \multicolumn{6}{|l|}{ Sente-se cobrado } \\
\hline Sim & $3,3(9)$ & 0,377 & 0,177 & 0,805 & 0,012 \\
\hline Não & $96,7(267)$ & 1 & & & \\
\hline \multicolumn{6}{|l|}{ Carga horária adequada } \\
\hline Sim & $72,5(200)$ & 0,981 & 0,731 & 1,315 & 0,896 \\
\hline Não & $27,5(76)$ & 1 & & & \\
\hline \multicolumn{6}{|l|}{ Sente-se seguro } \\
\hline Sim & $68,8(190)$ & 1,258 & 0,954 & 1,659 & 0,104 \\
\hline Não & $31,2(86)$ & 1 & & & \\
\hline \multicolumn{6}{|l|}{ Estresse } \\
\hline Sim & $33,3(92)$ & 0,303 & 0,185 & 0,495 & 0,000 \\
\hline Não & $66,7(184)$ & 1 & & & \\
\hline Exaustão (ISSL) & & & & & \\
\hline Sim & $92,8(256)$ & 0,488 & 0,389 & 0,612 & 0,000 \\
\hline Não & $7,2(20)$ & 1 & & & \\
\hline Horas de sono & $100(276)$ & 0,898 & 0,814 & 0,989 & 0,02 \\
\hline $\mathrm{X}^{2} \mathrm{RV}$ & & & $106,7(18) \mathrm{p}<$ & & \\
\hline Deviance & & & $187,4(257) \mathrm{p}$ & & \\
\hline
\end{tabular}

Fonte: Elaborada pelos autores 


\begin{tabular}{|c|c|c|c|c|c|}
\hline \multirow{3}{*}{ Variáveis } & \multirow{3}{*}{$\%(n)$} & \multicolumn{4}{|c|}{ Modelo ajustado } \\
\hline & & \multirow{2}{*}{$\mathbf{R P}$} & \multicolumn{2}{|c|}{ IC95\% } & \multirow{2}{*}{ p-valor } \\
\hline & & & Limite inferior & Limite superior & \\
\hline \multicolumn{6}{|l|}{ Sexo } \\
\hline Feminino & $49,60(137)$ & 0,574 & \multirow[t]{2}{*}{0,384} & \multirow[t]{2}{*}{0,858} & \multirow[t]{2}{*}{0,007} \\
\hline Masculino & $50,40(139)$ & 1 & & & \\
\hline \multicolumn{5}{|l|}{ Com quem reside } & \multirow{6}{*}{0,357} \\
\hline Família & $64,9(179)$ & 0,803 & 0,485 & 1,331 & \\
\hline Parentes & $7,2(20)$ & 1,251 & 0,666 & 2,352 & \\
\hline Amigos & $13,4(37)$ & 0,963 & 0,555 & 1,671 & \\
\hline Residência universitária & $14,5(40)$ & 1 & & & \\
\hline \multicolumn{5}{|l|}{ Sozinho } & \\
\hline \multicolumn{5}{|l|}{ Ensino médio } & \multirow{4}{*}{0,51} \\
\hline Privada & $37,30(103)$ & 0,94 & 0,463 & 1,907 & \\
\hline Pública & $61,20(169)$ & 0,75 & 0,369 & 1,526 & \\
\hline Misto & $1,40(4)$ & 1 & & & \\
\hline \multicolumn{5}{|l|}{ Atendimento psicológico } & \multirow{3}{*}{0,021} \\
\hline Sim & $49,60(137)$ & 0,645 & 0,443 & 0,937 & \\
\hline Não & $50,40(139)$ & 1 & & & \\
\hline \multicolumn{5}{|l|}{ Lazer semanal } & \multirow{3}{*}{0,554} \\
\hline Sim & $15,60(43)$ & 1,133 & 0,75 & 1,71 & \\
\hline Não & $84,40(233)$ & 1 & & & \\
\hline \multicolumn{5}{|l|}{ Atividade física } & \multirow{3}{*}{0,984} \\
\hline Sim & $38(105)$ & 1,004 & 0,684 & 1,473 & \\
\hline Não & $62(171)$ & 1 & & & \\
\hline \multicolumn{5}{|l|}{ Carga horária adequada } & \multirow{3}{*}{0,132} \\
\hline Sim & $72,50(200)$ & 1,546 & 0,877 & 2,727 & \\
\hline Não & $27,50(76)$ & 1 & & & \\
\hline \multicolumn{5}{|l|}{ Sente-se seguro } & \multirow{3}{*}{0,491} \\
\hline Sim & $68,80(190)$ & 1,189 & 0,727 & 1,945 & \\
\hline Não & $31,20(86)$ & 1 & & & \\
\hline Horas de sono & $100(276)$ & 0,726 & 0,604 & 0,872 & 0,001 \\
\hline \multicolumn{5}{|l|}{ Atividades acadêmicas } & \multirow[b]{3}{*}{0,142} \\
\hline Sim & $72,50(200)$ & 0,762 & & 1,096 & \\
\hline Não & $27,50(76)$ & 1 & 0,53 & & \\
\hline Idade & $100(276)$ & 1,011 & 0,975 & 1,048 & 0,569 \\
\hline Período & $100(276)$ & 0,91 & 0,855 & 0,968 & 0,003 \\
\hline $\mathrm{X}^{2} \mathrm{RV}$ & & & $61,54(15) \mathrm{p}<0$ & & \\
\hline Deviance & & & $237,63(260) \mathrm{p}=$ & & \\
\hline
\end{tabular}

Fonte: Elaborada pelos autores

\section{DISCUSSÃO}

Este estudo teve como objetivo investigar a prevalência de sintomas depressivos, ansiosos e de estresse em estudantes de Medicina de uma universidade federal do Rio Grande do Norte. Há tempos a formação médica vem sendo associada ao desgaste emocional e à prevalência de estresse, ansiedade e depressão nos discentes ${ }^{21}$. Neste estudo, verificou-se a presença de estresse em $66,3 \%$ dos estudantes, sintomas ansiosos em $33,6 \%$ e sintomas depressivos em $28 \%$. Outras pesquisas brasileiras com estudantes de Medicina encontraram dados similares, como é o caso do estudo de Vasconcelos et al. ${ }^{7}$ realizado no curso de Medicina da Faculdade
Pernambucana de Saúde que verificou a prevalência de 34,3\% de sintomas ansiosos e $24,9 \%$ de sintomas depressivos.

O trabalho de Lima, Soares, Prado e Albuquerque ${ }^{22}$ realizado na Universidade Federal do Paraná encontrou a prevalência de estresse em 60,9\% dos estudantes de Medicina. Outro trabalho com estudantes de Medicina de duas faculdades do Tocantins encontrou uma prevalência um pouco menor, de $52 \%$ dos alunos com estresse. Em uma faculdade privada no sertão da Paraíba, encontrou-se uma elevada prevalência de $78,98 \%$ de estresse em estudantes de Medicina ${ }^{23}$.

O trabalho de Leão, Gomes, Ferreira e Cavalcanti ${ }^{24}$, realizado num 


\begin{tabular}{|c|c|c|c|c|c|}
\hline \multicolumn{6}{|c|}{ Tabela 4} \\
\hline \multirow{3}{*}{ Variáveis } & \multirow{3}{*}{$\%(\mathrm{n})$} & \multicolumn{4}{|c|}{ Modelo ajustado } \\
\hline & & \multirow{2}{*}{$\mathrm{RP}$} & \multicolumn{2}{|c|}{ IC95\% } & \multirow{2}{*}{ p-valor } \\
\hline & & & Limite inferior & Limite superior & \\
\hline \multicolumn{6}{|c|}{ Atendimento psicológico } \\
\hline Sim & $49,5(138)$ & 0,303 & 0,115 & 0,802 & \\
\hline Não & $50,5(141)$ & 1 & & & 0,016 \\
\hline \multicolumn{6}{|c|}{ Atividades acadêmicas } \\
\hline Sim & $72,8(203)$ & 1,641 & 0,29 & 9,277 & \\
\hline Não & $27,2(76)$ & 1 & & & 0,575 \\
\hline \multicolumn{6}{|c|}{ Atividades extras } \\
\hline Sim & $28,7(80)$ & 0,306 & 0,094 & 0,992 & \\
\hline Não & 71,3(199) & 1 & & & 0,048 \\
\hline $\mathrm{X}^{2} \mathrm{RV}$ & & & $12,61(4), \mathrm{p}<\mathrm{c}$ & & \\
\hline Deviance & & & $92,8(274), p=$ & & \\
\hline
\end{tabular}

Fonte: Elaborada pelos autores

centro universitário do Ceará com estudantes da área da saúde, aponta uma prevalência de $25,9 \%$ tanto para depressão e quanto para ansiedade nos estudantes de Medicina. Rollemberg ${ }^{25}$ destaca que, dos estudantes de Medicina da Universidade Federal de Sergipe, 25,87\% apresentaram algum grau de ansiedade e $24,47 \%$ de depressão. Essa variação nas taxas de prevalência pode ocorrer por causa de fatores regionais específicos, das diferenças no acesso a serviços de saúde, da metodologia de pesquisa empregada, bem como dos questionários utilizados para a pesquisa. Uma metanálise recente sobre problemas de saúde mental em estudantes de Medicina no Brasil identificou a prevalência de 49,9\% para estresse, $32,9 \%$ para ansiedade e $30,6 \%$ para depressão ${ }^{26}$, corroborando a proporção encontrada no estudo atual, no qual os sintomas de estresse foram mais prevalentes, seguidos dos sintomas de ansiedade e, por último, de depressão.

Um ponto importante é que a maioria dos estudantes que tinha sintomatologia ansiosa ou depressiva apresentou um grau leve da sintomatologia, dado também corroborado por estudos anteriores ${ }^{24,26}$. Isso indica que, apesar da sintomatologia presente, o comprometimento funcional desses estudantes não era elevado. Não incluímos neste estudo discentes que haviam trancado o curso ou que estavam em regime domiciliar, assim o nível de comprometimento pode ser enviesado pelo critério de que os estudantes estivessem em atividades curriculares.

Em relação ao estresse, a maioria dos sintomas apresentados estava relacionada a fatores psíquicos, com predominância da fase de resistência, resultado semelhante a outros trabalhos ${ }^{22}$. A fase de resistência está associada a falta de motivação, cansaço excessivo e dificuldades na atenção e memória, interferindo em aspectos fundamentais do cotidiano universitário e prejudicando o desempenho ${ }^{23}$. Nessa fase, a prevalência de sintomas em estudantes de Medicina tem sido associada a aspectos de personalidade reforçados pelo ambiente médico, como o alto nível de exigência pessoal, rigidez e perfeccionismo ${ }^{27}$.

$\mathrm{Na}$ fase de exaustão, a prevalência de estresse no grupo estudado se mostrou mais expressiva nos primeiros períodos do curso. Os sintomas severos de depressão também estavam predominantemente presentes nos alunos do primeiro ao quarto período e os de ansiedade nos do quinto ao oitavo período. Não é de admirar que os estudantes dos primeiros anos se sintam mais estressados por causa da elevada quantidade de demandas a serem geridas, em especial a elevada quantidade de conteúdos teóricos dissociados da prática clínica ${ }^{28}$. Os resultados parecem sugerir que, com o passar dos períodos, os estudantes passam a desenvolver estratégias para lidar com os fatores estressantes presentes nos primeiros períodos do curso $^{22}$. Em relação à presença de sintomas ansiosos mais proeminentes do quinto ao oitavo período, eles podem ocorrer em virtude da entrada no ciclo clínico, que se associa a novas demandas na relação aluno-paciente, como também à cobrança da manifestação do conteúdo e do saber clínico nas atividades práticas e ambulatoriais.

Quase todos os alunos declararam sentir-se cobrados pelo curso (98\%). Apesar de essa afirmação não ser indicativa de sintomatologia de estresse, ansiedade ou depressão, ela revela a alta exigência que a formação médica impõe aos alunos, favorecendo o risco de estresse. Embora as escolas médicas se esforcem no sentido de apoiar os alunos e atualizar as estratégias pedagógicas com o propósito de melhorar a formação, há indícios de que os estudantes de Medicina, em diferentes países, vivenciam sofrimentos elevados durante o curso com implicações no desempenho acadêmico e na saúde ${ }^{29}$. Nesse sentido, $72 \%$ dos estudantes afirmaram considerar a carga horária exigida pelo curso excessiva, confirmando a percepção de que a formação demanda excessivamente deles. Esses dados são alarmantes principalmente quando se considera que os estudantes de Medicina que sofrem algum estado de adoecimento psíquico sentemse culpados por compartilharem suas emoções e relutam em buscar e receber tratamento ${ }^{30}$.

O estresse na formação pode predispor os alunos a sintomas de ansiedade e depressão, em especial quando associados a outros fatores de vulnerabilidade $\mathrm{e}^{30}$. O presente estudo identificou fatores associados a cada uma das sintomatologias. Em relação aos sintomas depressivos, observouse uma relação com o estresse, em especial os sintomas de exaustão, e com "se sentir cobrado". Isso é esperado considerando que na exaustão o sujeito perde a capacidade de adaptar-se ao contexto, adoecendo de diferentes maneiras ${ }^{17,31}$. A relação entre estresse e depressão é clara, tendo

\begin{tabular}{l|l}
7 & REVISTA BRASILEIRA DE EDUCAÇÃ̃ MÉDICA \\
\hline $44(1):$ e $040 ; 2020$
\end{tabular} 
em vista que a continuidade de situações estressantes propicia condições de esgotamento ao indivíduo e a depressão.

Estudar em escolas públicas foi associado a sintomas mais severos de depressão. Esse dado pode corroborar os estudos que apontam que estudantes com menores condições socioeconômicas têm mais chances de apresentar depressão ${ }^{32}$. Além disso, é importante discutir que a inserção mais proeminente do estudante de escola pública no curso de Medicina no Brasil ocorreu após a política de cotas nas universidades federais do país, questão ainda muito polêmica, tendo em vista a perspectiva de opositores de que o sistema de cotas coloca em risco a manutenção da qualidade do ensino público e deixa de lado a meritocracia ${ }^{33}$.

Diante disso, as angústias dos estudantes cotistas representam estressores importantes para sintomatologia depressiva. A própria expressão "aluno cotista" é considerada pejorativa pelos estudantes, muitas vezes associada a menor competência e desempenho por colegas e professores, apesar de essa expectativa não ser comprovada pelos índices acadêmicos e de os alunos cotistas e não cotista apresentarem índices semelhantes ${ }^{34}$. O estigma enfrentado influencia a autoestima do estudante e sua efetiva inclusão. Os estudos de Oliveira ${ }^{35}$ e Lemos $^{36}$ apontam narrativas de estudantes cotistas de Medicina que apresentaram sintomatologia depressiva durante o curso. Esses dados sinalizam que estudar o ensino médio em escola pública pode ser um fator de vulnerabilidade para o desenvolvimento de depressão durante a formação médica.

Dormir menos horas foi associado tanto a maiores graus de sintomatologia depressiva quanto à ansiosa. É importante considerar que alterações nos hábitos de sono caracterizam ambos os transtornos ${ }^{37}$, sendo difícil identificar se a menor quantidade de horas dormidas ocorre por causa da sintomatologia ansiosa e/ou depressiva. Entretanto, há dados que indicam que maus hábitos de sono têm uma relação importante com queixas de saúde mental ${ }^{38}$. Além disso, Waqas, Khan, Sharif e Khalid ${ }^{39}$ encontraram uma associação entre estresse acadêmico e má qualidade do sono entre estudantes de Medicina, com uma porcentagem considerável de entrevistados dormindo menos de cinco horas por noite. Esses dados ressaltam a importância de ações voltadas para a educação sobre bons hábitos de sono e de gestão de tempo.

Ainda em relação aos sintomas ansiosos, ser mulher foi um fator associado à maior ansiedade. Outros estudos vêm apontando que as mulheres apresentam maior prevalência de transtornos ${ }^{28,30}$. De acordo com Wahed e $\operatorname{Hassan}^{40}$, as mulheres são mais vulneráveis ao estresse e à ansiedade do que os homens e estão mais propensas a ter maior carga de atividades e mais sintomas físicos. Isso pode ocorrer em virtude da cobrança social dos múltiplos papéis a serem desempenhados pela mulher, acompanhados pela necessidade de reafirmarem a sua competência em espaços liderados por homens, como também pelas alterações hormonais próprias do sexo feminino. Além disso, Oliveira et al. ${ }^{41}$ ressaltam que as mulheres podem ser mais conscientes dos seus sentimentos do que os homens e, por isso, expressam com maior clareza seus sintomas ${ }^{41}$. Assim, talvez os homens também tenham elevados níveis de ansiedade tanto quanto as mulheres, mas não expressem nos questionários pela falta de consciência do que os aflige.

O período do curso também foi associado aos níveis de ansiedade, sendo a presença de níveis mais elevados nos dois primeiros ciclos do curso. Como citado anteriormente, os primeiros anos do curso são estressantes em decorrência da transição do ensino médio ou cursinho para o curso superior, com a necessidade de gestão de uma série de responsabilidades. Entretanto, o trabalho de Mousa, Dhamoon, Lander e Dhamoon ${ }^{32}$ constatou que os veteranos apresentavam mais estresse quando comparados aos alunos do primeiro e segundo ano. Isso pode acontecer em decorrência do início das fases práticas dentro do hospital, bem como por causa do primeiro contato com demandas específicas da carreira médica. Kulsoom e $\mathrm{Afsar}^{30}$ verificaram grande estresse em alunos do primeiro ano e um pico de estresse, ansiedade e depressão nos alunos do quarto ano, antes do internato, possivelmente pelo fato de os estudantes rodarem em vários setores do hospital. A mudança de estilo de enfrentamento ao longo do curso, o contato com pacientes graves, a disputa por estágios e atividades extracurriculares e a tensão em relação ao mercado de trabalho e à residência representam possíveis decepções em relação à medicina e fatores de estresse antes do internato ${ }^{41}$. Nesse sentido, muitos estudantes se mostraram inseguros em relação ao futuro profissional e à inserção no mercado de trabalho $(68,5 \%)$. Isso ressalta a importância de os estudos incluírem a investigação dos possíveis gatilhos e fatores mantenedores de estresse em cada fase/ciclo da formação médica.

Os estudantes que já haviam passado por acompanhamento psicológico ou psiquiátrico ao longo da vida apresentaram maior grau de sintomatologia ansiosa e sintomas de estresse na fase de exaustão. Esse dado corrobora a perspectiva de que os estudantes que desenvolvem transtornos mentais na formação médica apresentam uma predisposição a tê-los e de que o sofrimento psíquico pode ser anterior à formação. Essa perspectiva explica essa predisposição de diferentes maneiras, como seleção de perfis específicos de estudantes pelos concorridos vestibulares de medicina, acúmulo de estresse oriundo da própria seleção para o curso e/ou presença de traços de personalidade como neuroticismo ou baixa inteligência emocional, apontados como preditores de ansiedade, depressão e estresse ${ }^{42,43}$. Considerar essa hipótese como totalmente verdadeira é admitir que a maior parte dos estudantes apresenta essa predisposição, o que não se justifica quando se comparam as estimativas da prevalência de sintomas com a população geral. Os estudantes que necessitem de maior apoio emocional devem ser identificados para a gestão das demandas. As instituições de nível superior precisam estar preparadas para dar apoio, em especial quando altas prevalências de estresse, ansiedade e depressão são observadas ${ }^{26}$.

Os resultados encontrados neste estudo têm implicações para o planejamento de intervenções destinadas aos estudantes de Medicina da UFRN. É evidente a necessidade do foco em intervenções que visem à redução de estresse em estudantes, como as práticas de mindfulness e relaxamento com o objetivo de redução de estresse e a promoção de prática de atividades físicas e ações que incentivem o manejo de tarefas e gestão de tempo. Além disso, é imprescindível ofertar apoio psicológico aos estudantes oriundos de escola pública e/ou às mulheres, bem como espaços de escuta e apoio para que eles possam elaborar os estigmas e desenvolver estratégias de enfrentamento ao longo da formação. Devem-se ainda promover ações que visem ao incentivo à melhoria do sono. Outro ponto é a necessidade de identificar alunos com vulnerabilidades emocionais para que sejam ouvidos, acompanhados e encaminhados para apoio psicológico. Isso pode ser feito por intermédio dos professores ou do Serviço de Apoio ao Estudante, por meio de entrevistas com os discentes do primeiro período a fim de acolhêlos, estabelecer vínculo e acompanhar os que possam apresentar histórico prévio de transtorno mental. 
Para o conhecimento dos autores, este foi o primeiro estudo a investigar a prevalência de sintomas de estresse, ansiedade e depressão em estudantes de Medicina do RN. Entretanto, ele possui limitações. Uma delas é o tamanho da amostra, o que compromete a generalização dos achados a todos estudantes de Medicina. Além disso, neste estudo participaram apenas estudantes de medicina da UFRN, de modo que os resultados encontrados se aplicam apenas a eles. Fazem-se necessários estudos que incluam outras instituições e que comparem cursos com metodologias de ensino diferentes.

\section{CONCLUSÕES}

Este estudo reforçou a preocupação existente na comunidade acadêmica acerca da maior incidência de sintomas depressivos, ansiosos e de estresse em estudantes de Medicina. Além disso, indicou fatores potenciais que aumentam a vulnerabilidade dos alunos. No entanto, esses sintomas, quando percebidos, podem ser manejados por meio de apoio e intervenções que ajudem os discentes a lidar com os desafios da formação.

Acredita-se, portanto, que as instituições de ensino superior devem se comprometer com o desenvolvimento integral dos seus estudantes, apresentando estratégias institucionais para o enfrentamento dessa realidade. Assim, programas de tutoria-mentoring podem ajudar os alunos a se sentir apoiados e acolhidos na sua trajetória acadêmica. Além disso, os currículos do curso de Medicina devem inserir a discussão sobre saúde mental do estudante e/ou profissional de saúde, de forma precoce, estimulando-os a reconhecer seus limites e desenvolver estratégias necessárias ao autocuidado. E, por fim, os serviços de apoio psicossocial ao estudante, diante da crise, devem oferecer atendimento psicológico e/ou psiquiátrico, contribuindo, dessa forma, para um melhor desempenho acadêmico, a redução de evasão escolar e um melhor sentimento de bem-estar.

Assim, destaca-se a responsabilidade das instituições de formação médica no cuidado e apoio destinados aos discentes, considerando a diversidade de suas demandas e necessidades.

\section{REFERÊNCIAS}

1. Malajovich N, Vilanova A, Frederico C, Cavalcanti MT, Velasco LB. A juventude universitária na contemporaneidade: a construção de um serviço de atenção em saúde mental para estudantes. Mental 2017;11(21):356-77.

2. Padovani R da C, Neufeld CB, Maltoni J, Barbosa LNF, Souza WF, Cavalcanti HAF et al. Vulnerabilidade e bem-estar psicológicos do estudante universitário. Rev. bras. ter. cogn. 2014;10(1):2-10.

3. Moreira S, Dutra E. As implicações existenciais do sofrimento psíquico na vida acadêmica do estudante. Inclusão no ensino superior: docência e necessidades educacionais especiais. Natal: EDUFRN; 2013. p. 235-46.

4. Moreira S da NT, Vasconcellos RL dos SS, Heath N. Stress in medical education: how to face this reality? Rev. bras. educ. méd. 2015;39(4):558-64.

5. Meleiro A, Cordeiro Q, Razzouk D, Lima M. Consequências do trabalho na saúde mental do médico: qual a realidade? In: Conselho Regional de Medicina do Estado de São Paulo. Trabalho e saúde mental dos profissionais de saúde. São Paulo: Cremesp; 2015.

6. Almeida BRD, Minervino JA. Prevalência de transtorno mental comum em graduandos de Medicina na Universidade Federal da
Paraíba. João Pessoa. Monografia (Graduação em Medicina) Universidade Federal da Paraíba. 2017.

7. Vasconcelos TC de, Dias BRT, Andrade LR, Melo GF, Barbosa L, Souza E. Prevalência de sintomas de ansiedade e depressão em estudantes de medicina. Rev. bras. educ. méd. 2015;39(1):135-42.

8. Abrão CB, Coelho EP, Passos LB da S. Prevalência de sintomas depressivos entre estudantes de Medicina da Universidade Federal de Uberlândia. Rev. bras. educ. méd. 2008;32(3):315-23.

9. Dutra E. Ideação e tentativa de suicídio entre estudantes de medicina da UFRN e profissionais de saúde da rede pública de Natal. In: Borges L de O. Os profissionais de saúde e seu trabalho. São Paulo: Casa do Psicólogo; 2005, p. 281-98.

10. Alves TC de TF. Depressão e ansiedade entre estudantes da área de saúde. Revista de Medicina 2014;93(3):101-5.

11. Costa EF de O, Santana YS, Santos ATR de A, Martins LAN, Melo EV de, Andrade TM de. Sintomas depressivos entre internos de medicina em uma universidade pública brasileira. Rev. Assoc. Méd. Bras. 2012;58(1):53-9.

12. Gandini R de C, Martins M do CF, Ribeiro M de P, Santos DTG. Inventário de Depressão de Beck-BDI: validação fatorial para mulheres com câncer. Psico-USF 2007;12(1):23-31.

13. Kendall PC, Hollon SD, Beck AT, Hammen CL, Ingram RE. Issues and recommendations regarding use of the Beck Depression Inventory. Cognit. ther. res. 1987;11(3):289-99.

14. Beck AT, Epstein N, Brown G, Steer RA. An inventory for measuring clinical anxiety: psychometric properties. J. consult. clin. psychol. 1988;56(6):893-7

15. Cunha JA. Manual da versão em português das Escalas Beck. São Paulo: Casa do Psicólogo; 2001.

16. Cunha J. Estudo dos pontos de corte do BDI e do BAI na versão em Português. In: 8. Congresso Nacional de Avaliação Psicológica. Porto Alegre, RS. 1999; Poster vol. 78.

17. Lipp MN. Manual do Inventário de Sintomas de Stress para Adultos de Lipp. São Paulo: Casa do Psicólogo; 2000.

18. Ramiro F de S, Lombardi Júnior I, Silva RC da, Montesano FT, Oliveira NR de, Diniz RE, et al. Investigation of stress, anxiety and depression in women with fibromyalgia: a comparative study. Rev. bras. reumatol. 2014;54(1):27-32.

19. Assis CL de, Silva APF, Souza Lopes M de, Silva P da CB, Oliveira Santini T de. Sintomas de estresse em concluintes do curso de psicologia de uma faculdade privada do norte do país. Mudanças 2013;21(1):23-8.

20. Rossetti MO, Ehlers DM, Guntert IB, Leme IFA de S, Rabelo IS, Tosi SMVD, et al. O Inventário de Sintomas de Stress para Adultos de Lipp (ISSL) em servidores da Polícia Federal de São Paulo. Rev. bras. ter. cogn. 2008;4(2):108-20.

21. Teh CK, Ngo CW, binti Zulkifli RA, Vellasamy R, Suresh K. Depression, anxiety and stress among undergraduate students: A cross sectional study. Open Journal of Epidemiology 2015;5(4):260-268.

22. Lima RL de, Soares MEC, Prado SN do, Albuquerque GSC de. Estresse do estudante de Medicina e rendimento acadêmico. Rev. bras. educ. méd. 2016;40(4):678-84.

23. Estrela Y da CA, Rezende ACC, Guedes AF, Pereira C de O, Sousa MNA de. Estresse e correlatos com características de

REVISTA BRASILEIRA DE EDUCAÇÃO MÉDICA

9 $94(1): \mathrm{e} 040 ; 2020$ 
saúde e sociodemográficas de estudantes de Medicina. CES med. 2018;32(3):215-25.

24. Leão AM, Gomes IP, Ferreira MJM, Cavalcanti LP de G. Prevalência e fatores associados à depressão e ansiedade entre estudantes universitários da área da saúde de um grande centro urbano do Nordeste do Brasil. Rev. bras. educ. méd. 2018;42(4):55-65.

25. Rollemberg G de SM. Avaliação da presença de sintomas de ansiedade e depressão em estudantes de Medicina da Universidade Federal de Sergipe - Campus Lagarto. Lagarto. Monografia (Graduação em Medicina) - Universidade Federal de Sergipe; 2018.

26. Pacheco JP, Giacomin HT, Tam WW, Ribeiro TB, Arab C, Bezerra IM, et al. Mental health problems among medical students in Brazil: a systematic review and meta-analysis. Braz. J. Psychiatry. 2017;39(4):369-78.

27. Querido IA. Estresse, personalidade e habilidades sociais de estudantes de Medicina no internato. Goiânia. Dissertação (Mestrado Profissional em Ensino da Saúde) - Universidade Federal de Goiás; 2014.

28. Puthran R, Zhang MW, Tam WW, Ho RC. Prevalence of depression amongst medical students: a meta-analysis. Med. educ. 2016;50(4):456-68.

29. Fares J, Al Tabosh H, Saadeddin Z, El Mouhayyar C, Aridi H. Stress, burnout and coping strategies in preclinical medical students. North American Journal of Medical Sciences 2016;8(2):75-81

30. Kulsoom B, Afsar NA. Stress, anxiety, and depression among medical students in a multiethnic setting. Neuropsychiatr. dis. treat. 2015;11:1713.

31. Chaves LB, Souza TF de, Silva MVC da, Oliveira CF de, Lipp MEN, Pinto ML. Estresse em universitários: análise sanguínea e qualidade de vida. Rev. bras. ter. cogn. 2016;12(1):20-6.

32. Mousa OY, Dhamoon MS, Lander S, Dhamoon AS. The MD blues: under-recognized depression and anxiety in medical trainees. PLoS ONE 2016;11(6):e0156554.

33. Teixeira LAS, Couto MO, Santos PVL. Ação afirmativa em uma escola médica no Brasil: perspectivas dos estudantes. Revista Triângulo 2018;11(3):129-138

34. Castro ATB de. Estudantes de cotas: um convite à reflexão. Textos contextos (Porto Alegre) 2006;5(1):1-14.

35. Oliveira SS de. Afiliação universitária: trajetórias de estudantes cotistas e não cotistas em cursos de alto prestígio social na Universidade Federal da Bahia. Salvador. Dissertação (Mestrado em Educação) Universidade Federal da Bahia; 2018.

36. Lemos IBD. Narrativas de cotistas raciais sobre suas experiências na universidade. Rev. bras. educ. 2017;22(71):e227161.

37. American Psychiatric Association. DSM-5: Manual diagnóstico e estatístico de transtornos mentais. Porto Alegre: Editora; 2014.
38. Orzech KM, Salafsky DB, Hamilton LA. The state of sleep among college students at a large public university. J. am. col. health 2011;59(7):612-9.

39. Waqas A, Khan S, Sharif W, Khalid U, Ali A. Association of academic stress with sleeping difficulties in medical students of a Pakistani medical school: a cross sectional survey. PeerJ 2015;3:e840.

40. Wahed WYA, Hassan SK. Prevalence and associated factors of stress, anxiety and depression among medical Fayoum University students. Alexandria Journal of Medicine 2017;53(1):77-84.

41. Oliveira GS de, Rocha CA, Santos BÉF dos, Sena IS, Favaro L, Guerreiro MC. Prevalência e fatores associados à depressão em estudantes de medicina da Universidade Federal do Amapá. Revista de Medicina e Saúde de Brasília 2017;5(3):186-99

42. Yusoff MSB, Pa MNM, Mey SC, Aziz RA, Rahim AFA. A longitudinal study of relationships between previous academic achievement, emotional intelligence and personality traits with psychological health of medical students during stressful periods. Educ. health 2013;26(1):39-47

43. Lean LL, Hong RYS, Ti LK. How the personalities of medical students at the National University of Singapore differ from those of the local non-medical undergraduate population: a cross-sectional study. Singap. med. j. 2018;59(12):656.

\section{CONTRIBUIÇÃO DOS AUTORES}

Deyvison Soares da Costa contribuiu em todas as fases do estudo, inclusive na elaboração da versão final do artigo. Natany de Souza Batista Medeiros contribuiu na análise dos dados e na elaboração da versão final do artigo. Rayane Alves Cordeiro contribuiu na coleta dos dados e na elaboração da versão final do artigo. Everton Frutuoso contribuiu na análise dos dados e na revisão da versão final do artigo. Johnnatas Mikael Lopes contribuiu na análise dos dados. Simone da Nóbrega Tomaz Moreira contribuiu como orientadora em todas as fases do estudo, inclusive no desenho da pesquisa e na elaboração da versão final do artigo.

\section{CONFLITO DE INTERESSES}

Os autores declaram não haver conflito de interesses neste estudo.

\section{ENDEREÇO PARA CORRESPONDÊNCIA}

Deyvison Soares da Costa (Graduando em Medicina), Universidade Federal do Rio Grande do Norte (UFRN) - Hospital Universitário Onofre Lopes (HUOL), Departamento de Medicina Clínica, Av. Nilo Peçanha, 620 - Petrópolis, Natal - RN, 59012-300.

E-mail: deyvcosta@gmail.com 